\title{
Iterative Learning Fault Estimation Design for Nonlinear System with Random Trial Length
}

\author{
Li Feng, Ke Zhang, Yi Chai, Shuiqing Xu, and Zhimin Yang \\ College of Automation, Chongqing University, Chongqing 400044, China \\ Correspondence should be addressed to Ke Zhang; smeta@163.com
}

Received 11 July 2017; Revised 13 September 2017; Accepted 24 September 2017; Published 23 November 2017

Academic Editor: Michele Scarpiniti

Copyright (c) 2017 Li Feng et al. This is an open access article distributed under the Creative Commons Attribution License, which permits unrestricted use, distribution, and reproduction in any medium, provided the original work is properly cited.

\begin{abstract}
An iterative learning scheme-based fault estimation observer is designed for a class of nonlinear systems with randomly changed trial length. This is achieved by presenting a state observer for monitoring the system state and an iterative learning law for fault estimation in the presence of imprecise system model. An average factor is defined to deal with the lack and redundancy in tracking information caused by random trial length. Via the convergence analysis, sufficient design conditions are developed for estimation of fault signal. The observer gains and iterative learning law indexes are computed by solving the proposed conditions under $\lambda$-norm constraints. Numerical examples are presented to demonstrate the validity, the effectiveness, and the superiority of this method.
\end{abstract}

\section{Introduction}

Due to the increasing demand for reliability and safety and acceptable performance of control engineering systems, faulttolerant control [1-4] and fault detection $[5,6]$ have become an attractive theory and application topic in the last decades. Fault estimation [7-11] is supplementary to give the exact information of faults, thereby helping to reconstruct fault signals. Hence, it is further needed for the purpose of active fault-tolerant control to maintain the normal performance of systems. Over the past decades, observer-based fault estimation $[12,13]$ has received considerable attention both in academic research and in industrial application domains. It has made significant progress and covered a wide scope of research issues such as linear systems [14], nonlinear systems [15], time-delay systems [16], uncertain systems [17], multiagent systems [18] and fuzzy systems [19].

Note that all the above-mentioned results are based on accurate system models. However, on the assumptions that the systems are complex in practical issues [13, 20], they display insufficiency when fault estimation needs a model of higher precision. Therefore, it is an irresistible trend to develop tools for fault estimation analysis and design of real industrial systems. Recently, many researchers pay a lot of attention on iterative learning scheme-based fault estimation design [21-23]. For example, fault detection and estimation for nonlinear system are considered in [21], in which a novel iterative learning scheme is applied to make full use of valid information in last iteration. The estimation problem of periodically occurring faults is investigated in [22] for a class of nonlinear time-varying systems with bounded disturbance and measurement noise. The paper [23] proposes a novel fault estimation algorithm based on iterative learning scheme which is presented for a class of timevarying discrete switched systems with arbitrary sequence. These research results show that iterative learning scheme can estimate fault signal well without accurate models. It represents an effective approach proposed to improve current performance of systems by utilizing the previous experience. Iterative learning scheme is an important branch of intelligent algorithm, especially for dynamical systems that execute the same task repeatedly with a given desired trajectory to be tracked as accurately as possible.

In fact, the majority of industrial systems are repetitive systems $[24,25]$. For instance, industrial robots and computer numerical control systems often execute tasks repeatedly; servo systems with instruction or reference of periodic signal do the same tasks time by time. However, to the best of the authors' knowledge, the existing iterative learning schemes designs for fault estimation are in finite time interval of 
each trial. This condition may limit the applicable situation of iterative learning scheme. In real areas, the trial length may change because of some unexpected cases [26]. As skid phenomenon of conveyor belt occurs in factory assembly line, designated floors are different. As a summary of the foregoing, the trial lengths are randomly changed. It can be defined as two cases that it may be greater or smaller than the expected length with the change of iterations. Tracking information is missing if the operation length is smaller than the expected length while tracking information has redundancy if the operation length is larger than the expected length. Apparently, the mentioned traditional iterative learning schemes are compromised to deal with the problem of lack or redundancy in tracking information.

The aforementioned problems motivate the work in this paper: a novel iterative learning scheme-based fault estimator is designed to consider fault estimation problem for nonlinear systems with randomly changed trial length. Specifically, an average factor is defined to deal with the lack or redundancy in tracking error and state estimating error. Upon the convergence analysis of the proposed method, the states of the error system and the fault estimating error are proven to be uniformly ultimately bounded. Compared with the existing results, the main contributions of this paper are highlighted as follows:

(1) The proposed method using iterative learning scheme considers the state error information and fault estimating information from previous iteration to improve the fault estimation performance in the current iteration. In the existing approaches [29-31], only the state and output error in current iteration are considered in the fault estimating law and they are designed under the assumption that the system model is accurate. Different from these methods, the proposed method is free of precise system model and only needs bounds of parameter matrices.

(2) By using average factor, a novel iterative learning method is proposed for the first time to track the fault signal and to decrease the effect of lack and redundancy caused by randomly changed length. In [21-23], iterative learning observers are proposed for nonlinear systems, nonlinear time-delay system, and nonlinear systems with uncertainties. However, these methods are based on unchanged operation length. Hence, they are difficult to achieve the fault estimating performance of systems with randomly changed length.

(3) The proposed method inherits the advantages of conventional iterative learning scheme and the bounds of the faults and their derivatives could be unknown. That is, it can overcome the problem of randomly changed trial lengths without using advanced algorithms such as neural network and fuzzy system. As a result, the proposed approach can broaden the application scenario of iterative learning scheme in engineering practice.

The rest of this paper is organized as follows. The problem formulation and nonlinear repeated system with randomly

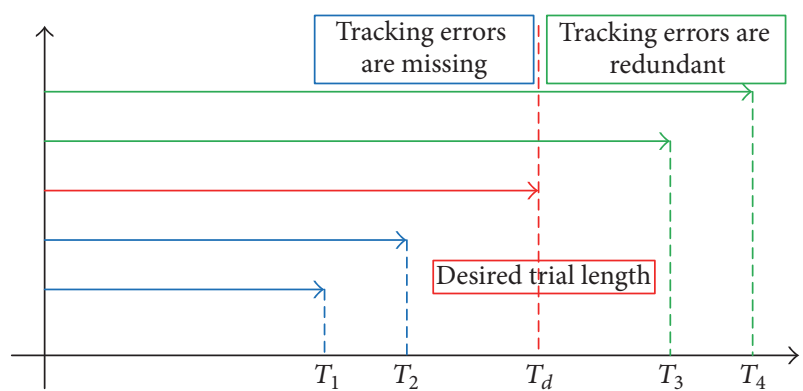

FIgURE 1: Randomly changed trial length.

changed trial length are introduced in Section 2. In Section 3, fault estimation using iterative learning scheme and average factor is proposed to achieve desired fault estimation results. Then, convergence analysis based on $\lambda$-norms is used to solve the problem in Section 4. Simulation results are presented in Section 5, and conclusion remarks are in Section 6.

\section{Problem Formulation}

Consider a class of nonlinear repetitive systems described by

$$
\begin{aligned}
& \dot{x}_{k}(t)=g\left(x_{k}(t), t\right)+B(t) u_{k}(t)+E(t) f_{k}(t), \\
& y_{k}(t)=C(t) x_{k}(t),
\end{aligned}
$$

where $k$ represents iterative index, $t \in\left[0, T_{k}\right]$ denotes operating time interval of system, $T_{k}$ is the time length in $k$ iterations, and $T_{d}$ is the desired time length. Meanwhile, $x_{k}(t) \in \mathfrak{R}^{n}, u_{k}(t) \in \mathfrak{R}^{m}, f_{k}(t) \in \mathfrak{R}^{m}$, and $y_{k}(t) \in \mathfrak{R}^{1}$ denote state, input, fault, and output of system (1), respectively. Further, $B(t), E(t)$, and $C(t)$ are time-varying matrices with appropriate dimensions and $C(t) B(t) \neq 0$.

Remark 1. The matrices $B(t), E(t)$, and $C(t)$ are time-varying in the proposed method, and it will be illustrated by theoretical derivation. However, constant matrices will be used in simulation parts for demonstrating the effectiveness of the proposed approach clearly and easily.

For the nonlinear term $g\left(x_{k}(t), t\right)$, there exists a known positive constant parameter $M_{g}$ which leads to satisfying the Lipschitz conditions (2) at any time $t \in\left[0, T_{d}\right]$ in any iterations $k \in N$ :

$$
\begin{gathered}
\left\|g\left(x_{k+1}(t), t\right)-g\left(x_{k}(t), t\right)\right\| \\
\leq M_{g}\left\|x_{k+1}(t)-x_{k}(t)\right\| .
\end{gathered}
$$

For system (1), the traditional fault estimation algorithm based on iterative learning scheme can achieve better performance of fault signal tracking and estimation in the case that the time interval is finite. However, it would not be the case that every trial ends in a fixed time of duration. Namely, the trial lengths are randomly changed with the increasing iterations. Evidently, conventional iterative learning schemes do not apply to these cases. Since the tracking errors are missing or redundant with nonuniform trial length (see Figure 1), it is hard to guarantee the validity of fault estimation. 
Based on the discussion above, a novel iterative learning scheme-based fault estimating law is proposed to deal with the missing or redundant problem caused by randomly changed iterative lengths in this paper. For expression to be concise, the following assumption and definition are given.

Definition 2. Define that there exists time constant $T_{i}$ that represents the interval factor in $i$ th iteration:

$$
P\left[t \leq T_{i}\right]= \begin{cases}0, & t \in\left[0, T_{d}-N_{1}\right) \\ p(t), & t \in\left[T_{d}-N_{1}, T_{d}+N_{2}\right] \\ 1, & t \in\left(T_{d}+N_{2},+\infty\right),\end{cases}
$$

where $N_{1}$ and $N_{2}$ are positive constant value, $N_{1} \in\left[0, T_{d}\right)$, and $N_{2} \in[0,+\infty)$. Let a stochastic variable $\gamma_{i}(t), t \in T_{i}$, satisfy Bernoulli distribution and tracking binary values 1 and 0 , in which $\gamma_{i}(t)=1$ stands for the event that process (1) can continue to the time instant in the $i$ th iteration and the probability of occurrence is $p(t), p(t) \in(0,1]$, while $\gamma_{i}(t)=0$ is the event that cannot last to $t$ and the probability of occurrence is $1-p(t)$ :

$$
P\left[\gamma_{i}(t)=1\right]=P\left[T_{i} \geq t\right]=1-P\left[T_{i} \leq t\right] .
$$

Define that $P\left[T_{i}=t\right]=0$; then one can obtain the occurrence probability of trial length $q(t)$ :

$$
q(t)= \begin{cases}1, & t \in\left[0, T_{d}-N_{1}\right) \\ 1-p(t), & t \in\left[T_{d}-N_{1}, T_{d}+N_{2}\right] \\ 0, & t \in\left(T_{d}+N_{2},+\infty\right) .\end{cases}
$$

Definition 3. For unified expression, define average factor Aer $\{\bullet\}$ as follows:

$$
\operatorname{Aer}\left\{Z_{i}(t)\right\}=\frac{1}{m} \sum_{j=1}^{m} Z_{i-j+1}(t), \quad t \in\left[0, T_{d}\right],
$$

where $k \geq m \geq 1$ is a given value and $Z_{i}(t), t=1,2, \ldots, m$, is a sequence that plays a pivotal role in the proposed fault estimator.

Lemma 4 (see [28]). The following inequality holds if there exists a positive constant parameter $\lambda>0$ :

$$
\sup _{t \in\left[0, T_{d}\right]}\left(e^{-\lambda t} \int_{0}^{t}\|x(\tau)\| d \tau\right) \leq \frac{1}{\lambda}\|x(t)\|_{\lambda} .
$$

Relevant evidential derivation can be obtained by the relationship between $\|*\|$ norm and $\|*\|_{\lambda}$ norm.

\section{Iterative Learning Scheme-Based Fault Estimation Design}

In order to ensure that the system output is equal to the actual output of the system, observer-based system prediction output is

$$
\begin{aligned}
\dot{\hat{x}}_{k}(t)= & g\left(\widehat{x}_{k}(t), t\right)+B(t) u_{k}(t)+E(t) \widehat{f}_{k}(t) \\
& +L(t)\left[y_{k}(t)-\widehat{y}_{k}(t)\right], \\
\hat{y}_{k}(t)= & C \widehat{x}_{k}(t) .
\end{aligned}
$$

In (8), $L(t)$ denotes the gain parameter of state observer and one should set it before estimation of system output. $\widehat{x}_{k}(t)$ is the estimate of the state vector, $\hat{y}_{k}(t)$ represents the estimate of the output vector, and $\widehat{f}_{k}(t)$ stands for the estimate of the fault signal.

Assumption 5. The initial state is equal to the expected state; that is, $\widehat{x}_{k}(0)=x(0)$.

Definition 6. According to system function (1) and system predicting function (11), the prediction error of system state is defined as

$$
e_{k}(t)=x_{d}(t)-\widehat{x}_{k}(t) .
$$

Then one can define the generalization error as

$$
e_{k}^{*}(t)=\gamma_{k}(t) e_{k}(t), \quad t \in\left[0, T_{d}\right] .
$$

On the one hand, it can be expanded to (11) when $T_{k}<T_{d}$ :

$$
e_{k}^{*}(t)= \begin{cases}e_{k}(t), & t \in\left[0, T_{k}\right] \\ 0, & t \in\left(T_{k}, T_{d}\right) .\end{cases}
$$
$T_{d}$

On the other hand, $e_{k}^{*}(t)$ can be expand to (12) when $T_{k} \geq$

$$
e_{k}^{*}(t)= \begin{cases}e_{k}(t), & t \in\left[0, T_{d}\right] \\ 0, & t \in\left(T_{d}, T_{d}+N_{2}\right] .\end{cases}
$$

Iterative learning scheme-based fault estimation approach contains system state predictive error. It is designed as

$$
\widehat{f}_{k+1}(t)=\operatorname{Aer}\left\{\widehat{f}_{k}(t)\right\}+\frac{m+1}{m} \sum_{j=1}^{m} \alpha_{k}(t) \times \dot{e}_{k-j+1}^{*}(t),
$$

where $\alpha_{k}(t)$ denotes design parameters and preset in fault estimation.

Remark 7. In practice, the probability distribution of the trial length could be estimated in advance based on previous multiple experiments or by experience. From (11), it can be seen that the average factor and parameter $1 \leq m \leq k$ are imported to eliminate the influence of lack and redundancy caused by randomly changed trial length. The value of $m$ depends on the empirical knowledge of experts. The performance of the proposed method is better when the results in $m$ iterations have more fault estimating information and vice versa. 
Definition 8. The associativity of actual fault signal and design (11) defines the fault estimating error term that is shown in (12):

$$
r_{k}(t)=f_{d}(t)-\widehat{f}_{k}(t)
$$

The expected fault signal $f_{d}(t)=f_{k}(t)$, since fault reconstruction and estimation are the highlights in this paper.

\section{Convergence Analysis}

The following theorem gives the convergence analysis of the proposed fault estimation approach using iterative learning scheme for nonlinear systems with randomly changed trial length.

Theorem 9. Consider the nonlinear systems described in (1) and fault estimation approach based on iterative learning scheme (13), and Assumption 5 holds. If the design parameters $\alpha_{i}(t)$ and $L(t)$ satisfy the following inequality:

$$
\sup _{t \in\left[0, T_{d}\right]}\left\|I-\alpha_{k}(t) p(t) E(t)\right\|=\mu_{1}<I,
$$

then the fault estimating error will converge to zero as iteration index $k$ goes to infinity, which is shown in

$$
\lim _{k \rightarrow \infty}\left\|r_{k}(t)\right\|_{\lambda}=0
$$

Remark 10. Based on the definition of $\lambda$-norm, one can see that the performance of proposed method is relevant to the parameter $\lambda$. The larger the value of $\lambda$, the faster the convergence rate of the iterative learning observer-based fault estimating approach.

Remark 11. Theorem 9 is obtained by assuming that the probability distribution $p(t)$ is known. However, one can use the lower and upper bounds of $p(t)$ to prove the convergence of the proposed method when $p(t)$ is unknown instead of expectation.

Proof. With Definition 6 and system function (1), let $\Delta g_{k}(t)=$ $g(\bullet)-\widehat{g}(\bullet)$; then $\dot{e}_{k}(t)$ is computed as

$$
\dot{e}_{k}(t)=\Delta g_{k}(t)+E r_{k}(t)-\mathrm{LC}_{k}(t) .
$$

Based on Definition 8, one can obtain that

$$
\begin{aligned}
& r_{k+1}(t)=f_{d}(t)-\frac{1}{m} \\
& . \sum_{j=1}^{m}\left[\widehat{f}_{k-j+1}-(m+1) \alpha_{k}(t) \gamma_{k-j+1}(t) \dot{e}_{k-j+1}^{*}(t)\right] \\
& =\frac{1}{m} \\
& . \sum_{j=1}^{m}\left[r_{k-j+1}(t)-(m+1) \alpha_{k}(t) \gamma_{k-j+1}(t) \dot{e}_{k-j+1}^{*}(t)\right] \\
& =\operatorname{Aer}\left\{r_{k}(t)\right\}-(m+1) \alpha_{k}(t) \operatorname{Aer}\left\{\dot{e}_{k}^{*}(t)\right\} .
\end{aligned}
$$

By the definition of average factor, $\operatorname{Aer}\left\{r_{k+1}(t)\right\}$ can be rewritten as

$$
\operatorname{Aer}\left\{r_{k+1}(t)\right\}=\frac{1}{m+1}\left[r_{k+1}(t)+m \operatorname{Aer}\left\{r_{k}(t)\right\}\right] .
$$

Then substituting (17) into the right side of (18), it can be computed out that

$$
\operatorname{Aer}\left\{r_{k+1}(t)\right\}=\operatorname{Aer}\left\{r_{k}(t)\right\}-\alpha_{k}(t) \operatorname{Aer}\left\{\dot{e}_{k}^{*}(t)\right\} \text {. }
$$

Let $\Xi\{\bullet\}$ denote the expectation of the stochastic variable; combining with (17) and (20), one can obtain that

$$
\begin{aligned}
\Xi\{\operatorname{Aer} & \left.\left\{r_{k+1}(t)\right\}\right\} \\
= & {\left[I-\alpha_{k}(t) p(t) E\right] \Xi\left\{r_{k}(t)\right\} } \\
& -\alpha_{k}(t) p(t) \Xi\left\{\operatorname{Aer}\left\{\Delta g_{k}(t)-\mathrm{LC}_{k}(t)\right\}\right\} .
\end{aligned}
$$

Based on Newton-Leibniz formula, (17) can be rewritten as

$$
e_{k}(t)=\int_{0}^{t} \Delta g_{k}(\tau)+\operatorname{Er}_{k}(\tau)-\mathrm{LC}_{k}(\tau) d \tau .
$$

Substituting $e_{k}(0)=0$ into (20), combining with the Lipchitz conditions of nonlinear term and taking Euclidean norm of both sides of (20), it can be obtained that

$$
\left\|e_{k}(t)\right\|=\int_{0}^{t}\left\|\left(M_{g}-\mathrm{LC}\right) e_{k}(\tau)\right\|+\left\|E r_{k}(\tau)\right\| d \tau \text {. }
$$

According to Bellman-Gronwall inequality, one has

$$
\left\|e_{k}(t)\right\| \leq M_{e} \int_{0}^{t} e^{M_{l c}(t-\tau)}\left\|r_{k}(\tau)\right\| d \tau,
$$

where $M_{e} \geq \sup _{t \in\left[0, T_{i}\right]}\|E\|$ and $M_{l c} \geq \sup _{t \in\left[0, T_{i}\right]}\left\|M_{g}-\mathrm{LC}\right\|$.

It follows by convergence analysis of fault estimating error $r_{k}(t)$. Substituting (24) into (21), the following inequality will be obtained:

$$
\begin{aligned}
& \left\|\Xi\left\{\operatorname{Aer}\left\{r_{k+1}(t)\right\}\right\}\right\| \\
& \leq\left\|\left[I-\alpha_{k}(t) p(t) E\right]\right\|\left\|\Xi\left\{\operatorname{Aer}\left\{r_{k}(t)\right\}\right\}\right\| \\
& \quad-\left\|\alpha_{k}(t) p(t)\right\| M_{l c} M_{e} \int_{0}^{t} e^{M_{l c}(t-\tau)} r_{k}(\tau) d \tau .
\end{aligned}
$$

Applying the $\lambda$-norm to both sides of (25), multiplying both sides with $e^{-\lambda t}, \lambda>0$, and according to Lemma 4 , one can conclude that

$$
\begin{aligned}
\left\|\Xi\left\{\operatorname{Aer}\left\{r_{k+1}(t)\right\}\right\}\right\| \leq & \mu_{1}\left\|\Xi\left\{\operatorname{Aer}\left\{r_{k}(t)\right\}\right\}\right\|_{\lambda} \\
& -\mu_{3} \frac{1-e^{\left(\mu_{3}-\lambda\right) T_{k}}}{\lambda-\mu_{3}}\left\|r_{k}(\tau)\right\|_{\lambda},
\end{aligned}
$$

in which $\mu_{1}=\sup _{t \in\left[0, T_{k}\right]}\left\|\left[I-\alpha_{k}(t) p(t) E\right]\right\|, \mu_{2}=$ $\sup _{t \in\left[0, T_{k}\right]}\left\|\alpha_{k}(t) p(t) M_{l c} M_{e}\right\|$, and $\mu_{3}=\max \left\{\mu_{2}, M_{l c}\right\}$.

Notice that the parameter $\mu_{1} \in[0,1)$ and the parameters $M_{e}$ and $M_{l c}$ have boundedness. Therefore, $\mu_{0}=\mu_{1}-\mu_{3}(1-$ 
$\left.e^{\left(\mu_{3}-\lambda\right) T_{k}}\right) /\left(\lambda-\mu_{3}\right)<1$ holds if it takes a big enough value of $\lambda$; that is, $\left\|r_{k+1}(t)\right\|_{\lambda} \leq \mu_{0}\left\|r_{k}(t)\right\|_{\lambda}$ holds. Herein one has

$$
\lim _{k \rightarrow \infty}\left\|r_{k}(t)\right\|_{\lambda}=0 \text {. }
$$

Simultaneously, noting (24), it can be obtained that

$$
\lim _{k \rightarrow \infty}\left\|e_{k}(t)\right\|_{\lambda}=0 .
$$

This is the end of Theorem 9 in proof.

\section{Illustrative Example}

This section is dedicated to showing the validity of the proposed new design methodology. The conservatism of the proposed method considered in this paper is evaluated numerically. For this, nonlinear systems are considered to demonstrate the effectiveness of the fault estimation approach using iterative learning scheme.

5.1. Constant Example. To illustrate the validity of the proposed method, a nonlinear MISO system described by (1) is considered in this subsection; that is,

$$
\begin{aligned}
{\left[\begin{array}{c}
\dot{x}_{k}^{1}(t) \\
\dot{x}_{k}^{2}(t)
\end{array}\right]=} & {\left[\begin{array}{ll}
2 & 1 \\
3 & 5
\end{array}\right]\left[\begin{array}{l}
\sin \left(x_{k}^{1}(t)\right) \\
\sin \left(x_{k}^{2}(t)\right)
\end{array}\right]+\left[\begin{array}{c}
u(t) \\
2 u(t)
\end{array}\right] } \\
& +\left[\begin{array}{l}
f(t) \\
f(t)
\end{array}\right], \\
y_{k}(t)= & {\left[\begin{array}{ll}
1 & 2
\end{array}\right]\left[\begin{array}{l}
x_{k}^{1}(t) \\
x_{k}^{2}(t)
\end{array}\right] . }
\end{aligned}
$$

The actual fault signal is given as

$$
\begin{aligned}
f(t)=\sin \left(\frac{2}{5} \pi t\right)+\sin (2 \pi t)+\sin (5 \pi t), & \\
& t \in[0,2] .
\end{aligned}
$$

To realize the above fault estimating and output predicting objectives, the period is set to be $T_{d}=2$, and the estimating values of initial states $\widehat{x}_{k}^{1}(0)$ and $\widehat{x}_{k}^{2}(0)$ are the same as the actual initial states value $x_{k}^{1}(0)$ and $x_{k}^{2}(0)$. In order to better show fault tracking results in each iteration, the maximum value of absolute error $E_{k}$ is introduced to evaluate the effectiveness of fault estimating performance in different iterations. The definition of $E_{k}$ is shown as follows:

$$
E_{k}=\sup _{t \in\left[0, t_{k}\right]}\left|f(t)-\widehat{f}_{k}(t)\right| \text {. }
$$

In the simulations, the designed fault estimating approach needs to be discretized by assuming that the interval between samples is $0.001 \mathrm{~s}$. The parameters are chosen as $L(t)=\left[\begin{array}{l}2.1 \\ 7.9\end{array}\right]$ and $\alpha(t)=\left[\begin{array}{ll}0.5 & 0.23\end{array}\right]$. In addition, $p(t) \in[0,1]$ is a randomly changed constant value, $N_{1}=0.5$, and $N_{2}=0.8$. Obviously, substitute the parameters into (15), and one has

$$
\sup _{t \in\left[0, T_{d}\right]}\left\|I-\left[\begin{array}{ll}
0.5 & 0.23
\end{array}\right] p(t)\left[\begin{array}{l}
1 \\
1
\end{array}\right]\right\| \leq 1-0.73 p(t)<1 .
$$

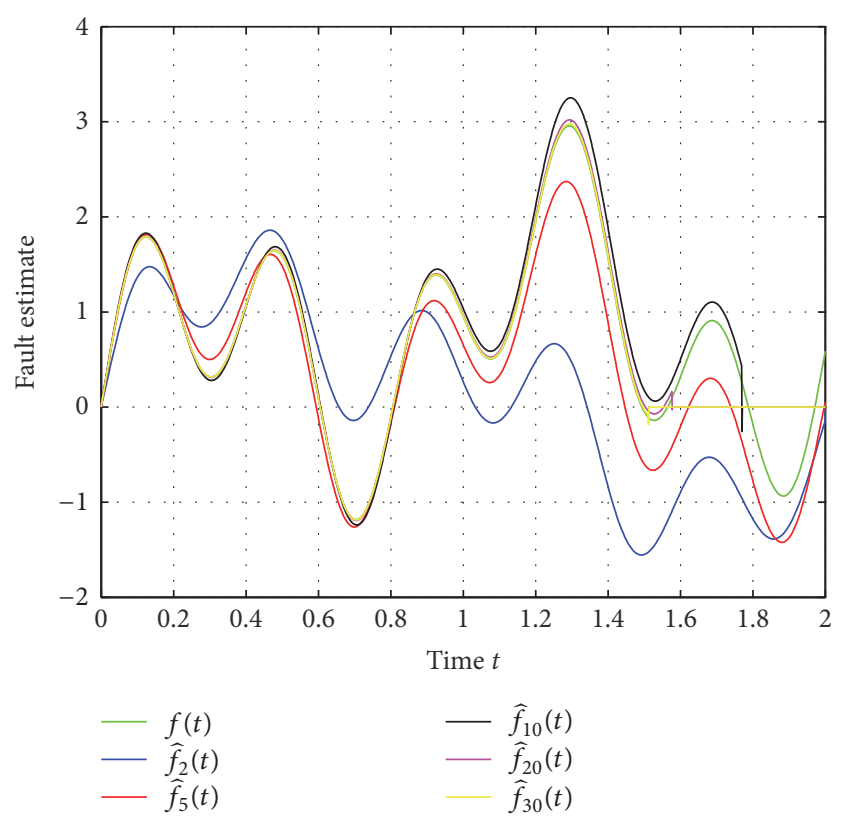

FIgURE 2: Fault estimating results in different iterations.

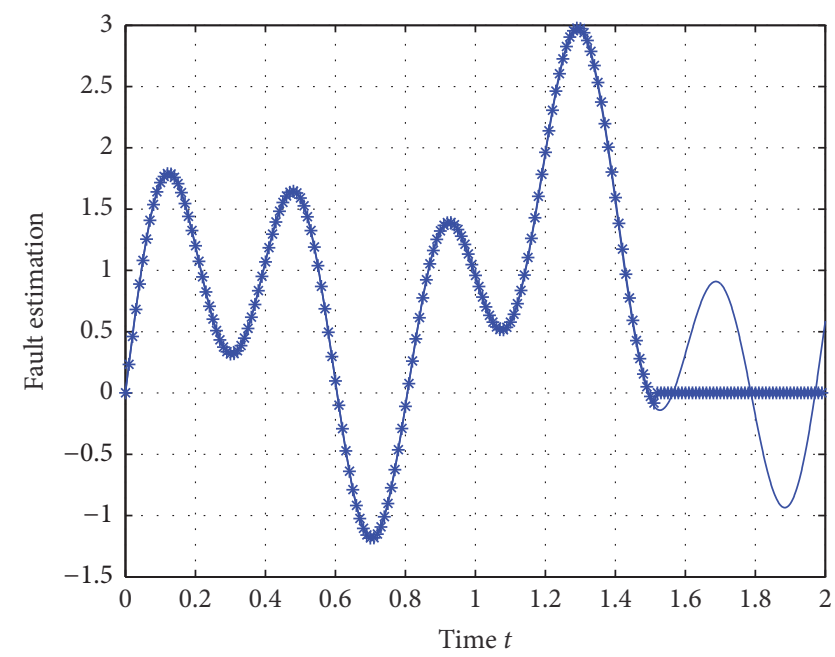

FIGURE 3: Fault estimating result in 30th iterations of constant system.

Namely, the selected parameters satisfy the conditions in Theorem 9. Simulation results on fault estimation using iterative learning scheme for nonlinear systems with randomly changed trial lengths are shown in Figures 2-4.

Figure 1 shows the fault estimating results and actual fault signal, in which $f(t), \widehat{f}_{2}(t), \widehat{f}_{5}(t), \widehat{f}_{10}(t), \widehat{f}_{20}(t)$, and $\widehat{f}_{30}(t)$ represent expected fault signal and estimated fault signals at second, fifth, tenth, twentieth, and thirtieth iterations, respectively. It is not hard to find out the difference of fault estimating result at different iterations. The operation time changes at different iterations and the tracking error decreases. The estimated fault signal at thirtieth iterations is plenty of overlap with the expected fault signal, which is shown in Figure 2. The variation trend of maximum absolute 


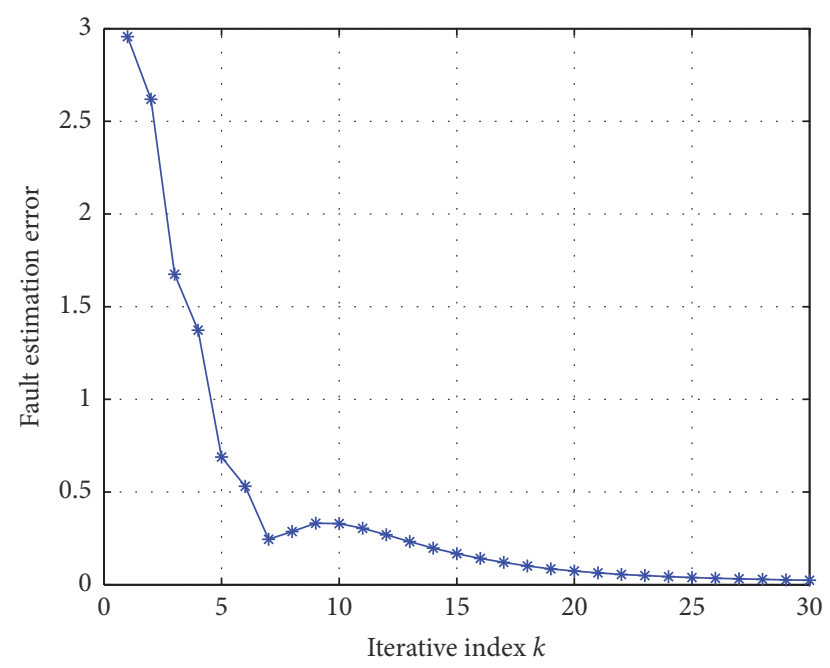

FIgURE 4: The variation trend of $E_{k}$ in constant system.

error $E_{k}$ is shown in Figure 2. It can be seen that $E_{k}$ decreases with iterations increase and converges to zero.

5.2. Time-Varying Example. In this section, the numerical time-varying example with faults is represented in the following form:

$$
\begin{aligned}
{\left[\begin{array}{c}
\dot{x}_{k}^{1}(t) \\
\dot{x}_{k}^{2}(t)
\end{array}\right]=} & {\left[\begin{array}{cc}
1 & 1 \\
3 & 5-\sin (t)
\end{array}\right]\left[\begin{array}{l}
\sin \left(x_{k}^{1}(t)\right) \\
\sin \left(x_{k}^{2}(t)\right)
\end{array}\right] } \\
& +\left[\begin{array}{c}
(1+\sin (t)) u(t) \\
2 u(t)
\end{array}\right] \\
& +\left[\begin{array}{c}
\left(1-e^{-t}\right) f(t) \\
f(t)
\end{array}\right], \\
y_{k}(t)= & {\left[\begin{array}{ll}
1-\cos (t) & 2
\end{array}\right]\left[\begin{array}{l}
x_{k}^{1}(t) \\
x_{k}^{2}(t)
\end{array}\right] . }
\end{aligned}
$$

The actual fault signal is given as

$$
f(t)= \begin{cases}\sin (4 \pi t), & t \in[0,1) \\ 2 t, & t \in[1,1.5) \\ 0, & \text { others. }\end{cases}
$$

One can chooses the parameters $L(t)=\left[\begin{array}{c}-1.1 \\ 20\end{array}\right]$ and $\alpha(t)=$ $\left[\begin{array}{ll}0.8 & 0.18\end{array}\right]$, respectively. The probability $p(t) \in[0,1]$ is a randomly changed constant value, $N_{1}=0.5$, and $N_{2}=$ 0.8 . Then, substitute the parameters into (15), and it can be obtained that

$$
\sup _{t \in\left[0, T_{d}\right]}\left\|I-\left[\begin{array}{ll}
0.8 & 0.18
\end{array}\right] p(t)\left[\begin{array}{l}
1 \\
1
\end{array}\right]\right\| \leq 1-0.98 p(t)<1 .
$$

One can find out that the conditions in Theorem 9 are satisfied. The actual fault signal and its estimate are given

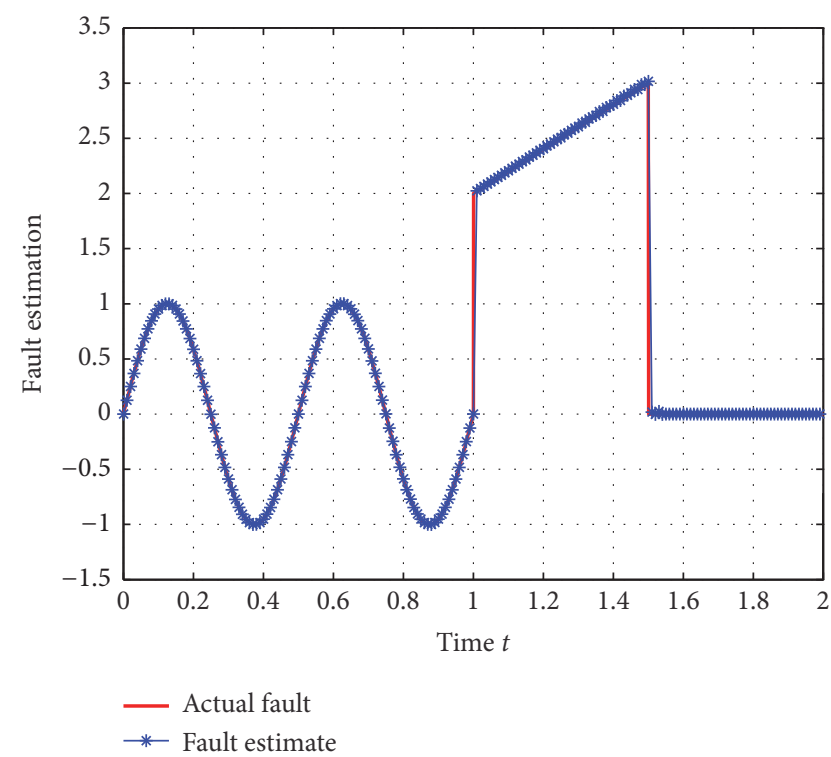

FIGURE 5: Fault estimating result in 30th iterations of time-varying system.

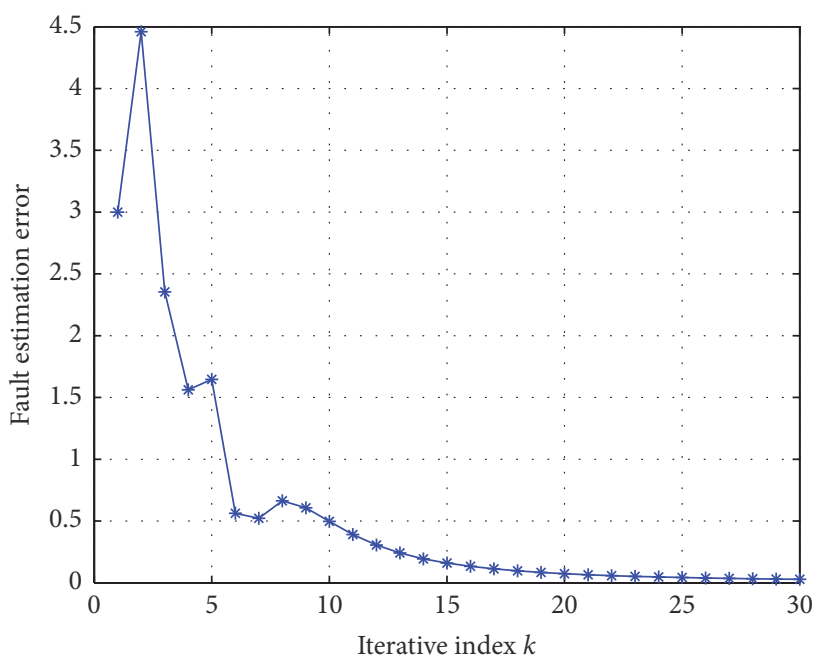

FIgURE 6: The variation trend of $E_{k}$ in time-varying system.

in Figure 5. And the estimating error result is displayed in Figure 6. The simulation results show a good estimation performance of fault signal.

Remark 12. According to the above results and discussion, it is obvious to find out that the proposed method is relevant to the bounds of system parameter matrices instead of the precise model of system. Meanwhile, it can be observed in Figures 2-6 that the proposed method can deal with different fault signals as well as lack and redundant problem caused by varying trial length. In other words, fault estimation using iterative learning scheme is excellent for fault estimation problem in nonlinear systems with random trial length.

5.3. Comparable Example. In this subsection, a class of nonlinear systems with equal trial length are employed to 


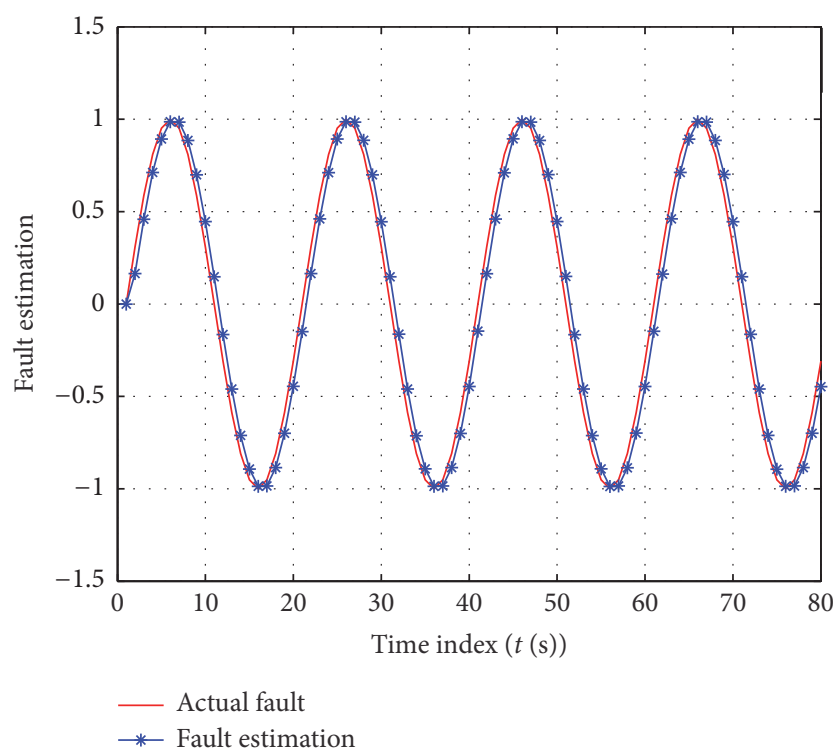

FIgURE 7: The fault estimation result in [27].

illustrate the superiority of the proposed method. That is because there are no existing fault estimation results in nonlinear systems with random trial length.

Consider a satellite launch vehicle along the pitch axis shown in [27], and the system is described as

$$
\begin{aligned}
{\left[\begin{array}{c}
\dot{x}_{p 1} \\
\dot{x}_{p 2}
\end{array}\right]=} & {\left[\begin{array}{ll}
0 & 1 \\
0 & 0
\end{array}\right]\left[\begin{array}{l}
x_{p 1} \\
x_{p 2}
\end{array}\right]+\left[\begin{array}{c}
0.1 \sin \left(x_{p 1}\right) \\
0
\end{array}\right] } \\
& +\left[\begin{array}{c}
0 \\
2.94
\end{array}\right] u(t)+\left[\begin{array}{c}
0 \\
2.94
\end{array}\right] f(t), \\
y= & {\left[\begin{array}{ll}
1 & 0
\end{array}\right]\left[\begin{array}{l}
x_{p 1} \\
x_{p 2}
\end{array}\right], }
\end{aligned}
$$

where $x_{p 1}$ is the pitch angle, $x_{p 2}$ stands for the pitch rate, $u(t)$ is input signal, and $f(t)$ represents the actuator fault signal that is shown in

$$
f(t)=\sin (0.1 \pi t), \quad t \in[0,80] .
$$

Let parameters $u=-k y, k=0.5963, \delta_{2}=0.1$, and $T_{d}=4$ and initial state value $x_{k}(0)=\left[\begin{array}{ll}0 & 0\end{array}\right]^{T}$, initial estimating state value $\widehat{x}_{k}(0)=\left[\begin{array}{ll}0 & 0\end{array}\right]^{T}, w(t)$ is white Gaussian noise with $1 \times 1$ dimension and square 0.01 , and the sample time period is $T=$ 0.01 .

In [27], one can chooses the parameters $L(t)=\left[\begin{array}{c}8.3031 \\ 40.9091\end{array}\right]$ and $\alpha(t)=\left[\begin{array}{ll}0.5963 & 0.5963\end{array}\right]$, respectively. Figure 7 represents the fault estimation results in [27]. It can be found out that the fault tracking result has time delay.

Meanwhile, in this paper, the parameters are set as $L(t)=\left[\begin{array}{l}2.4088 \\ 2.4872\end{array}\right]$ and $\alpha(t)=\left[\begin{array}{ll}-0.2595 & 0.1669\end{array}\right]$, respectively. Obviously, the parameters satisfy inequality (15). And fault estimation result using the proposed method is shown in Figure 8. It can be seen that the fault is estimated with satisfactory accuracy. Namely, the proposed method has better performance for fault estimation than the approach in [27].

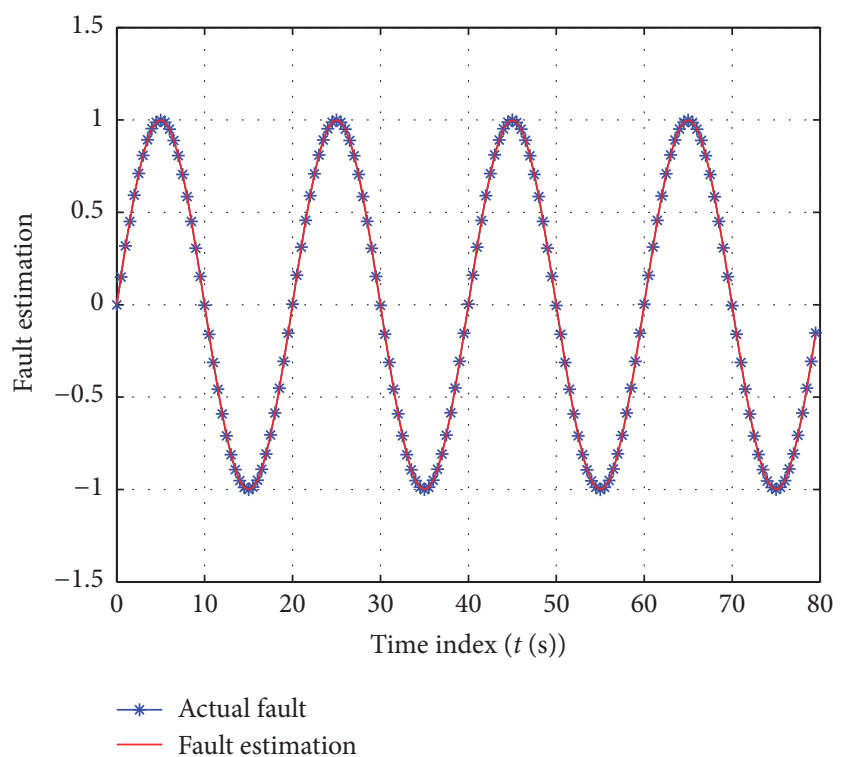

Figure 8: The fault estimation result in this paper.

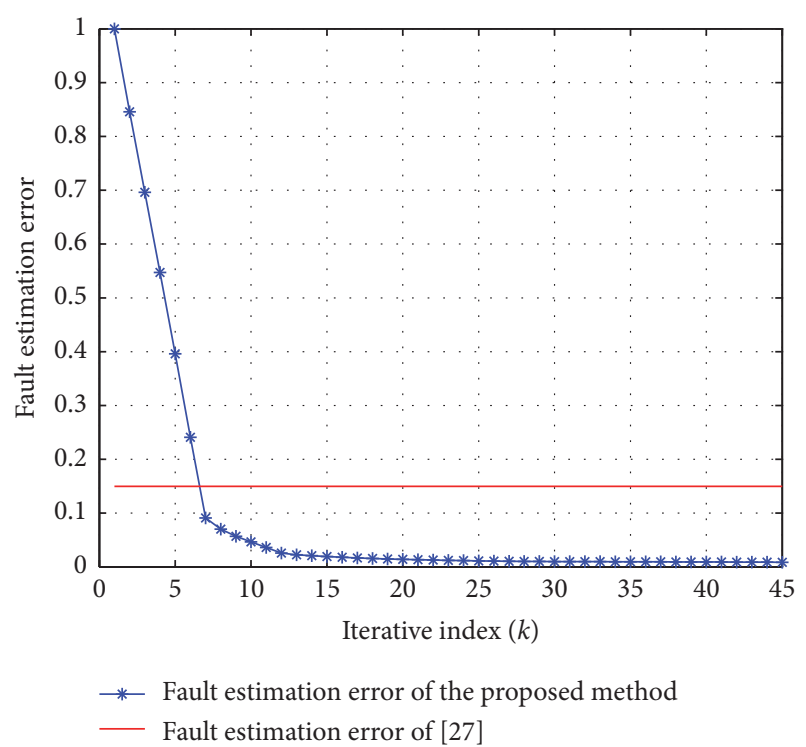

FIGURE 9: The comparison of fault estimation errors in [28] with the proposed method.

Moreover, the simulation comparison result of fault estimation is shown in Figure 9. In order to display the comparison result visually, maximum estimating error is chosen to decide the superiority. One can find out that the result in [27] is more precise than the proposed method in the first iterations. However, fault estimating result using iterative learning scheme is better than the existing method [27] after a few iterations.

Remark 13. From the simulation results (Figures 7-9), one can draw the conclusion that the proposed method based on iterative learning scheme is better than existing method [27]. The approach in this paper utilizes the state error 
information and fault estimating information in previous iteration to improve the fault estimation performance in the current iteration. Whereas the method in [27] ignores this experiential knowledge, only information in the current iteration is considered. As a result, the estimation error of the proposed method decreases with iteration increases until it converges to zero.

\section{Conclusion}

This paper proposes an iterative learning scheme-based fault estimator for nonlinear systems. The trial lengths are randomly changed which leads to missing and redundancy problems of tracking information. Firstly, an average factor is defined to deal with the information problem. Then, upon this definition, iterative learning scheme-based updating law is designed to estimate fault signal. Unlike conventional observer-based fault estimation methods, the proposed method considers the state error information and fault estimating information from previous iteration to improve the fault estimation performance in the current iteration. Further, the $\lambda$-norm method and mathematical induction are employed to obtain the convergence conditions of tracking errors. Specifically, all the parameter matrices are normbounded rather than the precise model in conventional methods. Hence, one can arrive at the conclusion that the proposed method broadens the application scenario of iterative learning scheme in engineering practice.

\section{Conflicts of Interest}

The authors declare that they have no conflicts of interest.

\section{Acknowledgments}

This work is supported in part by the National Natural Science Foundation of China $(61374135,61633005$, and 61673076).

\section{References}

[1] M. Blanke, "Diagnosis and Fault-Tolerant Control," IEEE Transactions on Automatic Control, vol. 49, no. 6, pp. 1037-1038, 2004.

[2] L. Liu, Z. Wang, and H. Zhang, "Adaptive NN fault-tolerant control for discrete-time systems in triangular forms with actuator fault," Neurocomputing, vol. 152, pp. 209-221, 2015.

[3] Z. Yang and Y. Chai, "A survey of fault diagnosis for onshore grid-connected converter in wind energy conversion systems," Renewable \& Sustainable Energy Reviews, vol. 66, pp. 345-359, 2016.

[4] J. Lan and R. J. Patton, "A new strategy for integration of fault estimation within fault-tolerant control," Automatica, vol. 69, pp. 48-59, 2016.

[5] L. Rong, X. Peng, and B. Zhang, "A reduced-order fault detection filtering approach for continuous-time markovian jump systems with polytopic uncertainties," Complexity, vol. 2017, Article ID 4927453, 14 pages, 2017.

[6] Y. Yang, S. Ding X, and L. Li, "On observer-based fault detection for nonlinear systems," Systems \& Control Letters, vol. 82, no. 1, pp. 18-25, 2015.
[7] B. Jiang and F. N. Chowdhury, "Fault estimation and accommodation for linear MIMO discrete-time systems," IEEE Transactions on Control Systems Technology, vol. 13, no. 3, pp. 493-499, 2005.

[8] M. Liu, X. Cao, and P. Shi, "Fault estimation and tolerant control for fuzzy stochastic systems," IEEE Transactions on Fuzzy Systems, vol. 21, no. 2, pp. 221-229, 2013.

[9] Z. Gao, "Fault estimation and fault-tolerant control for discretetime dynamic systems," IEEE Transactions on Industrial Electronics, vol. 62, no. 6, pp. 3874-3884, 2015.

[10] M. Witczak, M. Buciakowski, V. Puig, D. Rotondo, and F. Nejjari, "An LMI approach to robust fault estimation for a class of nonlinear systems," International Journal of Robust and Nonlinear Control, vol. 26, no. 7, pp. 1530-1548, 2016.

[11] Y. Song, J. Hu, D. Chen, D. Ji, and F. Liu, "Recursive approach to networked fault estimation with packet dropouts and randomly occurring uncertainties," Neurocomputing, vol. 214, pp. 340349, 2016.

[12] K. Zhang, B. Jiang, and P. Shi, "Fast fault estimation and accommodation for dynamical systems," IET Control Theory \& Applications, vol. 3, no. 2, pp. 189-199, 2009.

[13] S. Cheng, H. Yang, and B. Jiang, "An integrated fault estimation and accommodation design for a class of complex networks," Neurocomputing, vol. 216, pp. 797-804, 2016.

[14] S. M. Tabatabaeipour and T. Bak, "Robust observer-based fault estimation and accommodation of discrete-time piecewise linear systems," Journal of The Franklin Institute, vol. 351, no. 1, pp. 277-295, 2014.

[15] N. Oucief, M. Tadjine, and S. Labiod, "Adaptive observer-based fault estimation for a class of Lipschitz nonlinear systems," Archives of Control Sciences. Polish Academy of Sciences. Committee of Automatic Control and Robotics, vol. 26(62), no. 2, pp. 245-259, 2016.

[16] M. Kazerooni, A. Khayatian, and A. A. Safavi, "Robust delay dependent fault estimation for a class of interconnected nonlinear time delay systems," International Journal of Control, Automation, and Systems, vol. 14, no. 2, pp. 569-578, 2016.

[17] J. Zhang, A. K. Swain, and S. K. Nguang, "Robust sliding mode observer based fault estimation for certain class of uncertain nonlinear systems," Asian Journal of Control, vol. 17, no. 4, pp. 1296-1309, 2015.

[18] K. Zhang, G. Liu, and B. Jiang, "Robust unknown input observer-based fault estimation of leader-follower linear multiagent systems," Circuits Systems \& Signal Processing, pp. 1-8, 2016.

[19] K. Zhang, B. Jiang, and V. Cocquempot, "Fuzzy unknown input observer-based robust fault estimation design for discrete-time fuzzy systems," Signal Processing, vol. 128, pp. 40-47, 2016.

[20] P. Witczak, K. Patan, M. Witczak, and M. Mrugalski, "A neural network approach to simultaneous state and actuator fault estimation under unknown input decoupling," Neurocomputing, vol. 250, pp. 65-75, 2017.

[21] W. Cao, W. Cong, and M. Sun, "Iterative learning based fault detection and estimation in nonlinear systems," Journal of Systems Engineering and Electronics, vol. 23, no. 3, pp. 419-424, 2012.

[22] J. Shi, X. He, and D. Zhou, "Iterative learning based estimation of periodically occurring faults," IET Control Theory \& Applications, vol. 10, no. 2, pp. 244-251, 2016.

[23] W. Cao, Y. Guo, and M. Sun, "Fault estimation for discrete switched system based on iterative learning," Physics, vol. 63, no. 18, pp. 180202-1-180202-8, 2014. 
[24] S. Hara, Y. Yamamoto, T. Omata, and M. Nakano, "Repetitive control system: a new type servo system for periodic exogenous signals," Institute of Electrical and Electronics Engineers Transactions on Automatic Control, vol. 33, no. 7, pp. 659-668, 1988.

[25] L. Zhou and J. She, "Design of a robust output-feedback-based modified repetitive-control system," International Journal of Systems Science, vol. 46, no. 5, pp. 808-817, 2015.

[26] D. Shen, W. Zhang, and J.-X. Xu, "Iterative Learning Control for discrete nonlinear systems with randomly iteration varying lengths," Systems \& Control Letters, vol. 96, pp. 81-87, 2016.

[27] J.-W. Zhu, G.-H. Yang, H. Wang, and F. Wang, "Fault estimation for a class of nonlinear systems based on intermediate estimator," Institute of Electrical and Electronics Engineers Transactions on Automatic Control, vol. 61, no. 9, pp. 2518-2524, 2016.

[28] X. D. Li, T. W. S. Chow, J. K. L. Ho et al., "Iterative learning control with initial rectifying action for nonlinear continuous systems," Control Theory \& Applications, vol. 3, no. 1, pp. 49-55, 2009.

[29] B. Jiang, P. Shi, and Z. Mao, "Sliding mode observer-based fault estimation for nonlinear networked control systems," Circuits, Systems and Signal Processing, vol. 30, no. 1, pp. 1-16, 2011.

[30] S.-P. He, "Fault estimation for T-S fuzzy Markovian jumping systems based on the adaptive observer," International Journal of Control, Automation, and Systems, vol. 12, no. 5, pp. 977-985, 2014.

[31] Z. Wang, M. Rodrigues, D. Theilliol, and Y. Shen, "Actuator fault estimation observer design for discrete-time linear parametervarying descriptor systems," International Journal of Adaptive Control and Signal Processing, vol. 29, no. 2, pp. 242-258, 2015. 


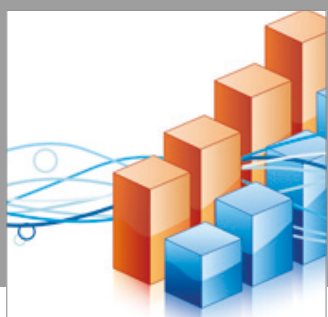

Advances in

Operations Research

vatersals

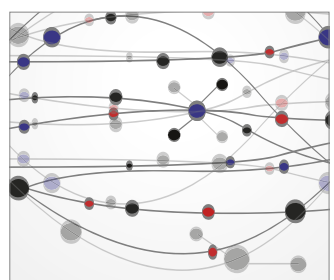

\section{The Scientific} World Journal
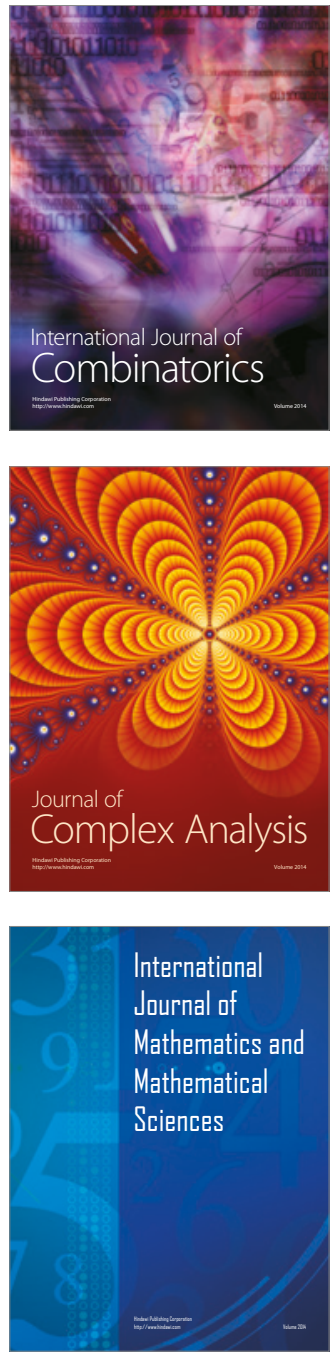
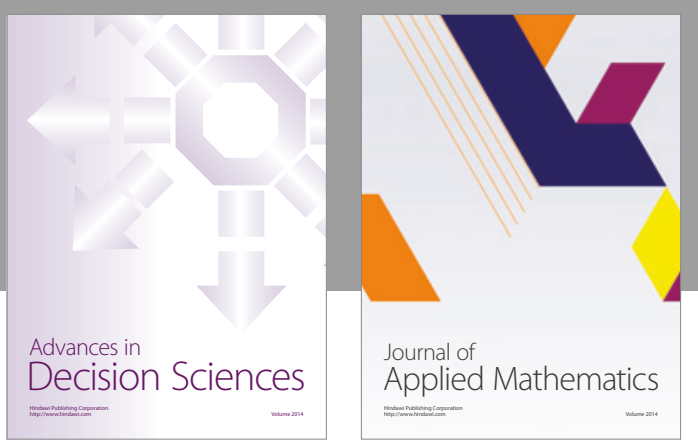

Algebra

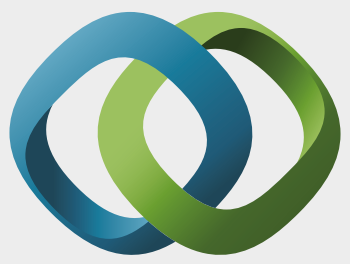

\section{Hindawi}

Submit your manuscripts at

https://www.hindawi.com
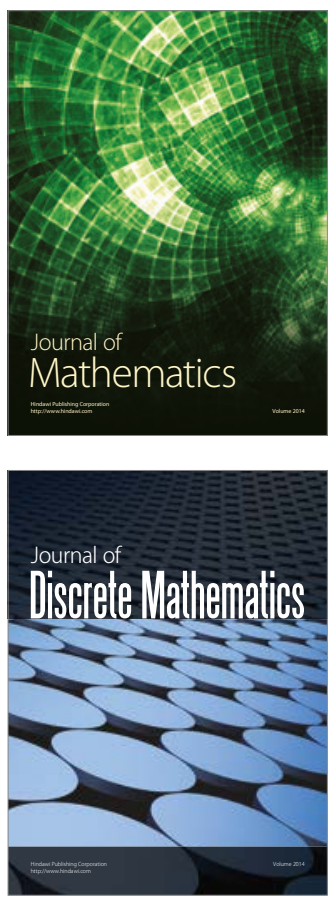

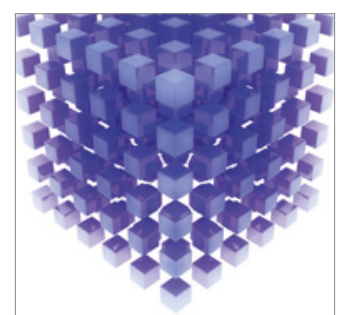

Mathematical Problems in Engineering
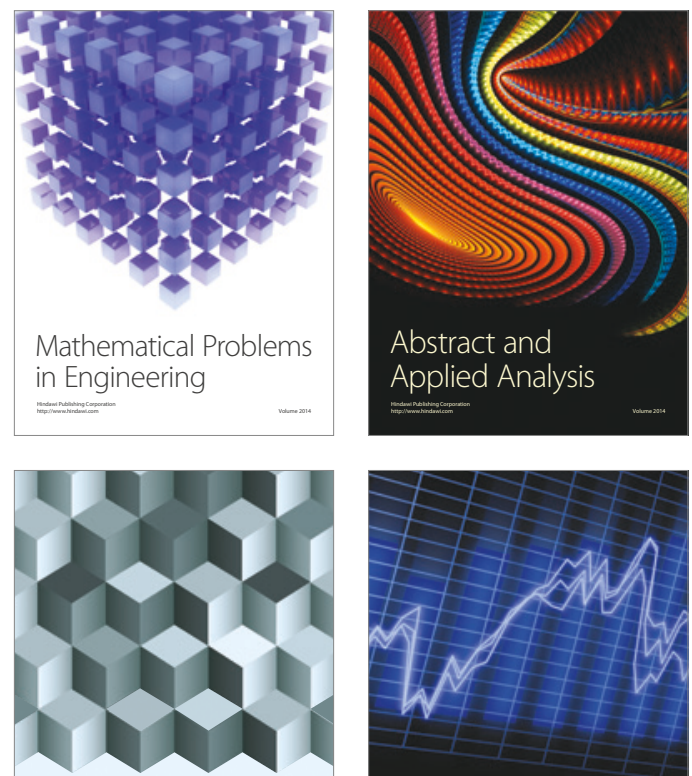

Journal of

Function Spaces

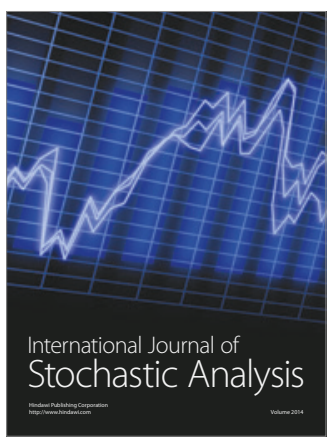

Probability and Statistics
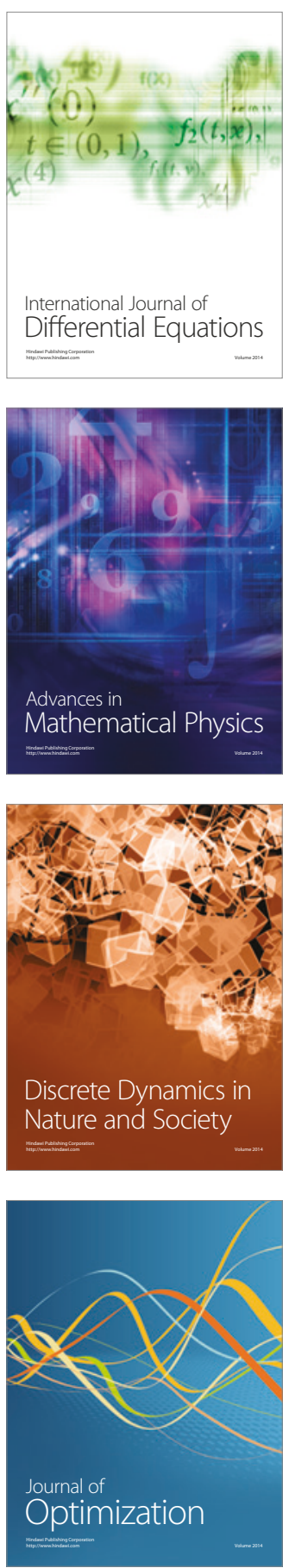